\title{
COMENTARIOS
}

\section{Por qué los Humedales de Puerto Viejo perdieron su protección legal: analizando los motivos}

\author{
Why the Wetlands of Puerto Viejo did loses its legal protection: analyzing the motives
}

\section{Dámaso W. Ramirez ${ }^{* 1,3}$ y Héctor Aponte ${ }^{2,3}$}

\author{
1 Área de Biología. Dirección de Cursos Básicos. Universidad Científica del Sur. Av. Antigua Carretera Panamericana Sur km 19 Villa El Salvador. - Lima, Perú. \\ 2 Facultad de Ciencias Veterinarias y Biológicas. Carrera de Biología Marina. Universidad Científica del Sur. Av. Antigua Carretera Panamericana Sur km \\ 19 Villa El Salvador. - Lima, Perú. \\ 3 Universidad Nacional Mayor de San Marcos, Museo de Historia Natural, Departamento de Dicotiledóneas. Avenida Arenales 1256, Jesús María, Lima-Perú. \\ * Autor para correspondencia \\ Email Dámaso Ramirez: dramirezh@cientifica.edu.pe; wilsonxviii@gmail.com \\ Email Héctor Aponte: haponte@cientifica.edu.pe
}

\section{Resumen}

Los humedales de Puerto Viejo son un ecosistema marino costero ubicado en el Sur de la ciudad de Lima. El año 2008, este ecosistema fue protegido por el estado y declarado Zona Reservada Humedales de Puerto Viejo, una categoría transitoria que brinda el SINANPE (Sistema Nacional de Áreas Naturales Protegidas por el Estado). En abril del 2017, una Resolución Ministerial declaró la desafectación de los Humedales de Puerto Viejo, perdiendo la categoría de Zona Reservada y quedando desprotegida legalmente. El presente trabajo analiza y discute los criterios técnicos de desafectación (representatividad, eficiencia, consistencia, conectividad, equilibrio, complementariedad y coherencia externa) que fueron aplicados a los Humedales de Puerto Viejo.

Palabras clave: Humedal; conservación; desierto costero peruano; Áreas Naturales Protegidas; Zona Reservada

\section{Abstract}

Puerto Viejo wetlands is a coastal marine ecosystem southern of Lima. Since 2008, this wetlands was protected by the Peruvian State and declared a Wetland Reserved Zone of Puerto Viejo, a transitory category provided by SINANPE (Sistema Nacional de Áreas Naturales Protegidas por el Estado). In April 2017, a Ministerial Resolution did reversal [desafectación] the transitory protection of Puerto Viejo Wetlands, losing the category of Reserved Zone and being legally unprotected. The present work analyzes and discusses the technical criteria for the reversal (representativeness, efficiency, consistency, connectivity, equilibrium, complementarity and external coherence) that were applied to Puerto Viejo Wetland.

Keywords: Wetlands; conservation; Peruvian coastal desert; protected natural areas; legal reserve area.

\section{Introducción}

Los humedales de Puerto Viejo son un ecosistema marino costero ubicado en el Sur de la ciudad de Lima, Provincia de Cañete, entre los distritos de San Antonio y Chilca. Diversos estudios realizados en este ecosistema han mostrado que el humedal cuenta con una diversidad representativa de organismos propios de la costa peruana (Iannacone et al. 2003, Paredes et al 2007, La Torre \& Aponte 2009, Tello \& Castillo 2010, Aponte
\& Cano 2013), y que además presenta una alta complementariedad con otros ecosistemas similares de la costa de Lima (Aponte \& Cano 2013).

El año 2008, este ecosistema fue protegido por el estado y declarado Zona Reservada Humedales de Puerto Viejo (Resolución Ministerial No 064-2008-AG), una categoría transitoria que brinda el SINANPE (Sistema Nacional de Áreas Naturales Protegidas por el Estado). Luego de una década de protección

Citación:

Ramirez D.W. \& H. Aponte. 2018. Por qué los Humedales de Puerto Viejo perdieron su protección legal: analizando los motivos. Revista peruana de biología 25(1): 049 - 054 (Febrero 2018). doi: http://dx.doi. org/10.15381/rpb.v25i1.14349

$\begin{array}{ll}\text { Presentado: } & 29 / 09 / 2017 \\ \text { Aceptado: } & 15 / 01 / 2018 \\ \text { Publicado online: } & 27 / 02 / 2018\end{array}$

Información sobre los autores:

DWR y HA desarrollaron la idea central del estudio, DWR escribió la primera versión del trabajo. Los autores no incurren en conflictos de intereses.

Journal home page: http://revistasinvestigacion.unmsm.edu.pe/index.php/rpb/index

(c) Los autores. Este artículo es publicado por la Revista Peruana de Biología de la Facultad de Ciencias Biológicas, Universidad Nacional Mayor de San Marcos. Este es un artículo de acceso abierto, distribuido bajo los términos de la Licencia Creative Commons Atribución-NoComercial-Compartirlgual 4.0 Internacional.(http://creativecommons.org/licenses/by-nc-sa/4.0/), que permite el uso no comercial, distribución y reproducción en cualquier medio, siempre que la obra original sea debidamente citadas. Para uso comercial, por favor póngase en contacto con editor.revperubiol@gmail.com. 
transitoria y múltiples acciones a favor de su conservación, en abril del 2017 se emitió una normativa legal que declara la desafectación de los Humedal de Puerto Viejo (Resolución Ministerial No 95-2017-MINAM), perdiendo la categoría de Zona Reservada (Z.R) y quedando desprotegida legalmente. El sustento técnico que se presentó (Informe No 638-2016 SERNANP-DDE) indica que la Z.R. Humedales de Puerto Viejo no cumple con las características básicas del componente físico establecidas por el SINANPE, las cuales son: representatividad, eficiencia, consistencia, conectividad, equilibrio, complementariedad y coherencia externa, referidas en el Plan Director de las Áreas Naturales Protegidas (D.S. 016-2009 MINAM). El informe de desafectación (Informe No 638-2016 SERNANP-DDE) evalúo las características básicas considerando una evaluación biológica rápida realizada en los Humedales de Puerto Viejo (Informe No1-2016).

Como consecuencia de esta medida de desafectación múltiples actores, como gobiernos regionales, municipales y organizaciones civiles han levantado su voz de protesta por esta decisión, sosteniendo que debilita las acciones de conservación que venían realizando para la protección del ecosistema. En este escenario socio-ambiental, el presente trabajo tiene el objetivo de analizar y discutir si los criterios técnicos de desafectación (representatividad, eficiencia, consistencia, conectividad, equilibrio, complementariedad y coherencia externa) fueron aplicados de forma adecuada en el Humedal de Puerto Viejo.

\section{Representatividad biológica}

Definición (D.S. 016-2009 MINAM): “Se entiende como representatividad biológica a los valores de la diversidad biológica en cuanto a los ecosistemas, especies y genes que existen en el país y que deben estar presentes en el SINANPE”. En el informe (638-2016 SERNANP-DDE) se indica que:

"El área contiene comunidades representativas de aves residentes, pero no de mamíferos ni de anfibios. En relación a la vegetación esta se encuentra representada en parches y con un alto porcentaje de especies introducidas. El área no aporta de forma significativa al SINANPE a cubrir el 10\% que se necesita conservar, todos los grupos taxonómicos no están representados, solo se reportan aves, además la vegetación se encuentra dispersa en parches".

A lo largo del documento se menciona que el humedal posee vegetación en parches. La vegetación que se presenta en el humedal no se encuentra de ninguna manera en parches, sino que más bien predomina un extenso pastizal, con dominancia de Distichlis y Salicornia, el cual tiene coberturas cercanas al 100\% (Aponte \& Ramírez 2011). Es verdad que la vegetación de algunas comunidades como el juncal y totoral tiene una distribución a manera de "parches", o para ser más preciso en forma de cinturones de vegetación que rodean los cuerpos de agua, lo cual es propio de estas comunidades vegetales, no fruto de una alteración antrópica de los patrones de distribución (Figura 1).

También se utiliza y resalta la presencia de especies introducidas en la vegetación como un indicador actual del estado del humedal. Aquí debemos considerar que la presencia de este grupo de especies en los humedales costeros es frecuente, cada vez que los mismos tienen interacción con la zona urbana (Aponte \& Cano 2013; Aponte et al. 2012; Ramirez et al. 2010). Así mismo, el porcentaje de especies introducidas en el humedal de
Puerto Viejo ha estado presente desde que el área fue propuesta como Zona Reservada (Expediente Técnico 2007) y según un análisis que hemos realizado considerando cuatro fuentes temporales (Expediente Técnico 2007, La Torre \& Aponte 2009, Informe 2012-SERNANP, Informe 01-2016-SERNANP), este porcentaje no ha cambiado considerablemente en el tiempo ( $27 \%$ en el $2007,27 \%$ en $2009,30 \%$ en el 2012 , 32\% en el 2016). En el área de estudio este grupo de especies introducidas se encuentran principalmente en la periferia del humedal o como rezagos de cercos vivos. En otras áreas protegidas como Pantanos de Villa y los Humedales de Ventanilla también existen especies introducidas, $28 \%$ y $35 \%$ respectivamente (Ramirez \& Cano 2010, Aponte \& Ramirez 2014) y en ninguno de estos casos ha sido un indicador para no conservar el área. Lamentablemente en dicho informe de desafectación no incluye los datos de las especies endémicas, en peligro, IUCN y CMS las cuales son de conservación prioritaria y son indicados en el documento de Evaluación Biológica Rápida de los Humedales de Puerto Viejo (Informe No1-2016). Estos datos justificarían apropiadamente la conservación de este ecosistema.

\section{Eficiencia}

Definición (D.S. 016-2009 MINAM): "Que satisfaga los objetivos propios de los sistemas de ANP con el menor costo de oportunidad posible. Se debe tratar de encontrar una adecuada relación entre el tamaño del sistema y la necesidad de satisfacer otros elementos básicos de bienestar social". El informe señala:

"El área no contribuiría de manera significativa al desarrollo del país, pero quizá lo hace de manera local, además los costos operativos para esta área serían mayores $(\$ 165 / \mathrm{ha})$ a los costos operativos de áreas de más de 100000 ha (\$2.45/ha)”.

En este punto debemos considerar que según el Plan Director de ANP (D.S. 016-2009), el establecimiento de ANP debe realizarse bajo un enfoque de costo-beneficio. En el informe solo se hace hincapié en los costos que generaría el área, pero no en los beneficios económicos que brindaría el humedal debido a su uso directo o indirecto. Ejemplos de usos directo con beneficios económicos podría darse a través de un pago por el ingreso al área para realizar un turismo sostenible como la observación de aves (Birdwatching) o como un ambiente natural para le educación ambiental de niños y jóvenes o como un "laboratorio natural"

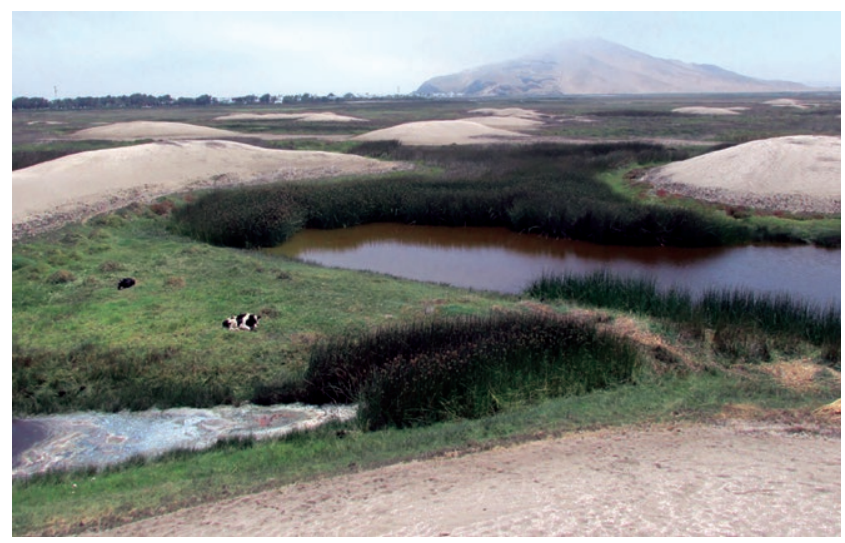

Figura 1. Los Humedales de Puerto Viejo (2016). Se observa un extenso pastizal (gramadal), dunas y los totorales y juncales alrededor de los cuerpos de agua distribuidos a manera de "parches". 
para la investigación. El humedal también brinda beneficios económicos a la población local y se da a través de la extracción de la totora balsa (Schoenoplectus californicus) y junco (Schoenoplectus americanus), de igual forma el humedal tiene un uso indirecto debido al servicio ambiental de captación de carbono. Un trabajo realizado en el área de estudio indica el gran potencial que tiene este humedal para captar carbono (Palomino \& Cabrera 2008) un servicio ecosistémico de alcance local y regional.

\section{Consistencia}

Definición (D.S. 016-2009 MINAM): "Las áreas naturales protegidas por el Estado deben tener la superficie suficiente para asegurar la continuidad de los procesos naturales de los ecosistemas, poblaciones mínimas viables o diversidad genética de las especies”. El informe indica:

"El área más que ecosistemas, contiene hábitats dispersos cada uno con sus particularidades donde las especies se desarrollan, pero a nivel de hábitats no ocurren procesos ecológicos que beneficien a otras poblaciones de especies fuera del humedal, favorecen sólo a las poblaciones que se encuentran allí; mostrando que el ecosistema contribuye localmente mas no contribuye a otras áreas del SINANPE. Las superficies definidas como unidades de vegetación presentes en el área no muestran la suficiente extensión, especialmente la zona arbustiva y la zona de juncal, debido a que se registran en parches".

En principio el texto muestra una confusión en el concepto y manejo de los términos hábitat y ecosistema llegando a considerarlos excluyentes. Un ecosistema viene hacer la conformación de una comunidad biótica en interacción con los factores abióticos allí presentes (Smith \& Smith 2007); el hábitat, por otro lado, se define como el lugar dónde vive un organismo (Di Bitetti 2012). Partiendo de estas definiciones los humedales de Puerto Viejo representa un gran ecosistema marino-costero que contiene múltiples hábitats como las diferentes comunidades vegetales o las lagunas circundantes, y todos ellos interactúan a través de procesos ecológicos relacionados a la dinámica del agua propia de estos ambientes. En los humedales costeros el agua es el elemento que interconecta los factores abióticos como el sustrato con los seres vivos como las comunidades vegetales, las cuales ayudan a formar el hábitat para una diversidad de fauna desde invertebrados hasta vertebrados (Young 1998) y decir que "El área, más que ecosistemas contiene hábitats dispersos..." es desconocer estos procesos y componentes elementales de estos ecosistemas.

El humedal al ser una fuente natural de agua dulce con múltiples hábitats interconectados representa un área de descanso y alimentación para poblaciones de aves migratorias que no son propias de Puerto Viejo como las "gaviotas de franklin" o "yanavico", y que representan migraciones latitudinales y altitudinales hacia el humedal. Incluso poblaciones de "yanavico" han llegado a establecerse en el humedal (Carlos 2008) lo cual demuestra que este ecosistema beneficia a poblaciones biológicas externas, contribuyendo local y regionalmente a conservar la biodiversidad. De igual forma, el área favorece la conservación de la biodiversidad para otras ANP, por su posición geográfica estratégica Puerto Viejo permite el nexo entre dos humedales costeros de importancia internacional (RAMSAR) como son el Refugio de Vida Silvestre Los Pantanos de Villa y La Reserva Nacional de Paracas, conformando un área de refugio, alimen- tación y descanso para las aves. De esta forma ayuda a mantener una conectividad ecológica entre humedales costeros, formando un corredor biológico en la costa central del Perú.

También se menciona que muchas especies externas al humedal no se benefician de la protección del área, sin considerar los beneficios obtenidos por los servicios ecosistémicos de depuración del agua y regulación del clima propios de humedales como este (Batzer \& Sharitz 2006), que aportarían a la conservación y conectividad de otras especies como reptiles y aves en los ecosistemas desérticos aledaños. Así mismo, el informe indica que las unidades de vegetación como el juncal no muestran la suficiente extensión por tener una distribución en "parches". En los humedales costeros se debe tener presente que los juncales tienen una distribución natural de esta forma, rodeando los cuerpos de agua, y representan una porción del área total en Humedales como Pantanos de Villa y Ventanilla.

\section{Conectividad}

Definición (D.S. 016-2009 MINAM): "Que los espacios que componen el sistema estén funcionalmente interconectados de manera que sean posibles los desplazamientos de individuos, los procesos de recolonización, los flujos genéricos y otros procesos ecológicos. Esto es particularmente importante cuando el tamaño individual de las ANP no es suficiente y la movilidad de las especies es alta”. El informe señala:

"Característica aplicable únicamente al grupo de aves que utilizan el humedal como lugar de paso durante su migración. No es un ecosistema utilizado por otras especies para conectarse con otros ecosistemas, debido a la naturaleza del mismo".

Se indica que "únicamente" lo utilizan el grupo de aves para su migración. En realidad, se trata de cientos de aves (gaviota de franklin) que utilizan estos ecosistemas durante su migración, y que sin la presencia de humedales como este, no tendrían zonas de descanso. Estudios realizados muestran que solo en un año son centenas de aves que la visitan, siendo Puerto Viejo, uno de los humedales con más especies de aves reportadas en Lima (Acuy Yánac \& Pulido 2008). Además, se llega a la conclusión de carencia de conectividad para otras especies sin algún tipo de estudio de conectividad.

Por otro lado, existen indicios que nos indicarían la importante conectividad que puede ocurrir en este ecosistema. En dos inventarios biológicos de biodiversidad en los Humedales de Puerto Viejo (Informe 2012-SERNANP, Informe 01-2016-SERNANP) se reporta especies silvestres de flora como Nolana humifusa, en los suelos arenosos del humedal, lo cual demuestra una conectividad con ecosistemas de lomas o tillandsiales de los alrededores, dónde suele encontrarse poblaciones de esta especie. De igual forma, otras especies arbustivas reportadas para el humedal como Baccharis salicifolia y Pluchea chingoyo son propias de suelos arenosos de las riveras de ríos costeños formando los ecosistemas conocidos como montes ribereños (Ferreyra 1983, Montesinos \& Mondragon 2013) y muestran que este humedal tendría una dinámica de conectividad con otros ambientes costeros.

\section{Equilibrio}

Definición (D.S. 016-2009 MINAM): "Que los Ecosistemas y poblaciones de especies estén contenido de manera equilibrada, 
sin que se dé redundancia de algunos de ellos e insuficiencia de otros". El informe menciona:

"Esta característica muestra insuficiencia en el área por ser un ecosistema con fuerte presión antrópica. Es una zona que se encuentra cerca a las playas, las que son muy concurridas por la población aledaña. Por tanto, debido a las actividades antrópicas que estaría generando desequilibrio en las poblaciones existentes en el ámbito de la Zona Reservada Humedales de Puerto Viejo"

Se indica como criterio para desafectarlo, que el área es insuficiente y que hay demasiada presión antrópica, cuando estas características no han sido obstáculos para conservar otros humedales como los Pantanos de Villa y Humedales de Ventanilla que tienen un área similar y también están afectados por presión antrópica (Ramirez \& Cano 2010; Aponte \& Ramirez 2014), y más bien su identificación ha llevado a tomar mejores medidas para su protección. Por ejemplo, la presión antrópica por urbanización ha ocasionado que los humedales de Ventanilla sufran un mayor impacto, perdiendo 78 ha, en comparación a los humedales de Puerto Viejo que solo han perdido 30 ha (Moschella 2012). Actualmente los humedales de Ventanilla están protegidos como Área de Conservación Regional y Puerto Viejo ha perdido su categoría de protección transitoria. Se menciona que el área tiene una fuerte presión antrópica por estar cerca a las playas que son concurridas por las poblaciones aledañas, en general esta presión afecta a la mayoría de los humedales costeros del Perú, incluyendo ANP como Pantanos de Villa y Paracas, así mismo esta visita ocurre principalmente en los meses de verano, dando una oportunidad para que los humedales y sus poblaciones biológicas se recuperen el resto de tiempo. Si bien la presión antrópica amenaza la conservación de humedales costeros insistimos que esta no puede ser un criterio para desafectar un área, sino más bien para tomar medidas que permitan protegerla.

Por otro lado, el informe presupone que hay desequilibrio ecosistémico en las poblaciones biológicas, pero no se señala en cuales poblaciones, ni se presentan estudios que lo respalden. Es cierto que la presión antrópica en humedales costeros ha originado la reducción y fragmentación de estos ecosistemas (Young 1998, Moschella 2012, Ramirez \& Cano 2010, Aponte \& Ramirez 2014) pero también es cierto que hay una respuesta o resiliencia de las poblaciones biológicas para adaptarse a estas presiones, por ejemplo, en los grupos de aves y vegetación presente (Kusch et al. 2008, Aponte \& Ramirez 2011).

\section{Complementariedad}

Definición (D.S. 016-2009 MINAM): "Que cada ANP aporte algo significativo y diferente al conjunto, asociado con el grado de intervención humana autorizado (categoría), pero independiente - en principio- del nivel político y de administración que le corresponda. Este enfoque de complementariedad reconoce que en el Sistema no existen áreas de segundo grado entre las ANP de carácter nacional y aquellas de carácter regional y privado”. El informe seńala:

"El ecosistema de esta zona Reservada, contiene hábitats con especies similares que no contribuyen a aumentar la riqueza de especies del SINANPE, pues ya se encuentran protegidas en otras áreas de conservación como por ejemplo los Manglares de Tumbes; y siendo un área pequeña, no se evidencian ecosistemas diversos (solo está el humedal) que contribuyan a complementar áreas de conservación colindantes y/o que contengan ecosistemas similares con poca extensión. Según la evaluación biológica rápida mostró resultados de especies introducidas, observándose especies propias de ambientes con presión antrópica, señalando además que no se evidenció especies representativas".

El informe presupone de manera apresurada que Puerto Viejo no contribuye a aumentar la riqueza de especies del SINANPE sin presentar un estudio básico de similitud con otros humedales que respalden esta conclusión, aduciendo además que los hábitats y las especies de Puerto Viejo ya se encuentran protegidos en los Manglares de Tumbes. En primer lugar, la comparación con los Manglares de Tumbes es inapropiada, en vista que es un tipo de humedal con una dinámica funcional y una flora y fauna diferente. En un análisis de similitud (Jaccard) entre la flora de Puerto viejo (Expediente Técnico 2007) y los Manglares de Tumbes (INRENA 2007) encontramos solamente un $9 \%$ de similaridad, lo cual demuestra lo diferente que son estos ecosistemas. Hasta cierto punto hubiera sido más coherente sustentar que las especies de Puerto Viejo ya se encontrarían en otras ANP como Pantanos de Villa debido a que son ecosistemas parecidos y geográficamente próximos. Sin embargo a pesar de lo anterior, en un estudio realizado en seis humedales de la costa central (Aponte \& Cano, 2013), se encontró que Pantanos de Villa y Puerto Viejo presentan una baja similitud (29\%) en la composición de especies vegetales, por lo cual la complementariedad entre estos humedales es alta (71\%). Por otra parte, se Indica que no hay especies representativas, cuando las especies representativas de la costa son el Junco, la Totora y la salicornia, presentes en el humedal según el Inventario Rápido de Diversidad Biológica en Humedales de Puerto Viejo (Informe No1-2016). Así mismo, este Inventario Rápido señala que si hay especies representativas en los grupos de plantas, aves y reptiles, señalando que solo en el grupo de mamíferos no se encontraron especies nativas.

Se menciona que Puerto Viejo es un área pequeña (275.81 ha), sin embargo, tiene una superficie similar a ANP como Pantanos de Villa (263.27 ha) o Humedales de Ventanilla (275.45 ha), además de incluir un conjunto de ecosistemas costeros (lagunas, cerros, desierto) y marinos (playa) según su expediente técnico de Zona Reservada (INRENA 2007). También se ha demostrado que humedales de menor tamańo son importantes para conservar la biodiversidad regional, proteger la conectividad ecológica y mantener las dinámicas de fuente-sumidero para poblaciones de aves y anfibios (Semlitsch \& Bodie 1998, Gibbs 2000).

\section{Coherencia externa}

Definición (D.S. 016-2009 MINAM): “Que los espacios naturales protegidos no se vean afectados negativamente por las actividades humanas que se realizan en la matriz territorial en que se inscriben, actuando además como espacios críticos para la salud y perdurabilidad del equilibrio ecológico general del territorio". El informe indica:

"Actualmente, en el área se registran derechos preexistentes, carreteras asfaltadas y trochas carrozables de uso constante que perturban las poblaciones de aves".

Se indica que el área tiene derechos preexistentes, carreteras y trochas, lo cual es cierto; sin embargo, esta es una característica de los humedales protegidos de la costa de Lima (Cano et al. 
1998; Ramirez et al. 2010; Ramirez \& Cano, 2010) que no amerita que se le quite la protección, sino más bien, nos lleva a tomar medidas para mejorar su situación. También se menciona que las aves son perturbadas de forma constante por estas estructuras, lo cual es correcto, pero también se ha demostrado que las aves tienen una rápida adaptación a estas perturbaciones antrópicas reflejando un uso dinámico de los hábitats y de los recursos presentes en humedales costeros (Kusch et al. 2008). Por lo cual, las aves podrían adaptarse a la presencia de la actividad antrópica si tenemos un manejo adecuado del hábitat (Riffell et al. 1996, Kusch et al. 2008).

\section{Comentarios finales}

A nivel legal, la protección de los humedales es aparentemente favorable, se cuenta con múltiples herramientas de gestión regional, nacional e internacional que permiten proteger estos ecosistemas. Por ejemplo, el Perú cuenta con una Estrategia Nacional de Humedales (D.S. 004-2015-MINAM) que representa el principal instrumento para la conservación de estos ambientes; asimismo, existen normas legales como el Decreto Supremo No 012-94-AG que declara intangible las áreas de los cauces, riberas y fajas marginales de los ríos, arroyos, lagos, lagunas y vasos de almacenamiento. A nivel internacional La Convención Ramsar (1971) protege a los humedales de importancia internacional y garantiza su uso racional. Todas estas herramientas legales permitirían proteger estos ecosistemas, sin embargo, en la práctica estas herramientas se ven omitidas y en muchos casos funcionan como leyes blandas (Mazuelo Bellido 2004) o careciendo de reglamentos claros y precisos para la sanción de quienes los incumplan.

El caso de desafectación de los Humedales de Puerto Viejo representa una medida legal (RM No 95-2017-MINAM) que menos cava los esfuerzos de conservación de los humedales costeros en el Perú, ya que ha dejado a este ecosistema sin ningún tipo de protección. Así mismo, ha sido una decisión unilateral sin considerar la opinión de los otros actores involucrados en la conservación de este ecosistema (como el Comité de Vigilancia de los Humedales de Puerto de Viejo, Municipalidad Distrital de San Antonio, Gobierno Regional de Lima) y que venían realizando medidas articuladas para la protección de este humedal. En la actualidad los Humedales de Puerto Viejo son un área natural sin protección legal, quedando a merced de perder más área o resultar más fragmentado por ambiciones de urbanismo, que viendo el escenario actual podrían aprovecharlo.

Los Humedales de Puerto Viejo fueron desafectados aplicando criterios que se sustentaron en la información brindada por una Evaluación Biológica Rápida (Informe No 1-2016) realizada en el área de estudio. Con el objetivo de verificar la información de este inventario biológico hemos revisado este documento y encontramos que el informe de desafectación incluye solo parte de la información, obviando otros datos importantes que apoyarían la conservación de este ecosistema. Dicho informe de desafectación no menciona los datos de especies endémicas, amenazadas, CMS, IUCN y CITES incluidos en la flora y fauna del humedal y que son indicados en el documento del inventario biológico (Informe No 1-2016). Por ejemplo, la especie vegetal Batis marítima es categorizada en Peligro Crítico (CR) por la normativa peruana y a nivel de Áreas Naturales Protegidas de la Costa Central no se encuentran en Pantanos de Villa, Ventanilla o Paracas, solo se ha registrado poblaciones pequeñas en los $\mathrm{Hu}-$ medales de Puerto Viejo. De igual forma este humedal presenta una comunidad biológica (totoral de Schoenoplectus californicus "totora balsa") casi extintas en humedales de la costa central, no estando presente por ejemplo en Pantanos de Villa, y cuya especie dominante además es utilizada desde épocas prehispánicas hasta la actualidad, para construir los famosos "caballitos de totora" en el norte del país, y en el área de estudio se utiliza para elaborar artesanías como tapetes y techos. Por otra parte, la Evaluación Biológica Rápida fue realizada solo en una temporada del año (marzo 2016) por lo que la información obtenida sobre la biodiversidad del humedal es parcial, pudiendo incrementarse si se hubiera realizado de forma anual. En el mismo documento se recomienda realizar evaluaciones de ápoca húmeda y seca con el fin de obtener resultados globales. De igual forma hubiera sido recomendable apoyarse en otros trabajos de investigación realizados en el área para saber el estado de la biodiversidad del humedal y tener un mejor sustento científico-técnico para aplicar los criterios de desafectación.

Con este trabajo quisiéramos poner en discusión, más que el uso del instrumento legal de desafectación, la aplicación inadecuada de los criterios técnicos establecidos para la desafectación del humedal. La R.M No 95-2017-MINAM declara la Desafectación de la Zona Reservada Humedales de Puerto Viejo, argumentando que el humedal; "no cumple con las características básicas del componente fisico del SINANPE, como son: representatividad, eficiencia, consistencia, conectividad, equilibrio, complementariedad y coherencia externa". De acuerdo al análisis que hemos realizado, argumentamos que tales criterios fueron aplicados de forma incorrecta, considerando solo parte de la información del inventario biológico del humedal (Informe No 1-2016) y empleando de forma equivocada conceptos como ecosistemas, hábitat y fragmentación "parches" en los humedales de Puerto Viejo.

El principal problema de los humedales de Puerto Viejo como Zona Reserva no es que su biodiversidad y el ecosistema no cumplan con las características básicas para ser un ANP, sino principalmente los derechos de terrenos preexistentes de propietarios privados que se superponen al área del humedal y que actualmente representan problemas judicializados que no permiten una adecuada zonificación. Esto sí representa un obstáculo para la viabilidad de la gestión y el costo para conservar un área en tales condiciones. Sin embargo, durante los últimos años que el humedal fue una Zona Reservada (Informe No 06-2016 SERNANP-ZRHPV/J) se produjo una sinergia de la población local, extractores del recurso vegetal, población del Distrito de San Antonio, de los clubes de playa circundantes y de algunos propietarios privados de contribuir a la protección y manejo de este ecosistema.

Finalmente, considerando la situación actual de los Humedales de Puerto Viejo es necesario que los diferentes actores involucrados en la conservación de este ecosistema (gobiernos regionales, municipales, asociaciones civiles, instituciones públicas especializadas como SERNANP y la comunidad científica) trabajemos en una estrecha colaboración para salvaguardarlo, tomando medidas concretas y rápidas para mejorar la situación legal y ambiental de este humedal. Consideramos que, si bien existen debilidades institucionales a nivel de ordenamiento territorial, es posible tomar medidas de protección como la creación de un Área de Conservación Regional, que antes ya ha sido una propuesta técnica para el humedal (ET-ZRHPV 2009), y que 
permitiría conservar la biodiversidad y los servicios ambientales que brinda este importante humedal costero.

\section{Agradecimientos}

Agradecemos a los gestores que nos han permitido adquirir los documentos técnicos citados en el presente estudio y que nos han involucrado en la problemática planteada en esta discusión. Así mismo, agradecemos a los revisores por sus valiosas sugerencias al manuscrito

\section{Literatura citada}

Acuy Yánac M., \& V. Pulido. 2008. Peru: informe anual. Censo Neotropical de Aves Acuáticas 2007 [en línea]. En D. Unterkofler \& Blanco (Eds.), El Censo Neotropical de Aves Acuáticas 2007; Una herramienta para la conservación. Buenos Aires, Argentina: Wetlands International. Recuperado a partir de http://lac.wetlands.org/Portals/4/CNAA $\% 20$ 2008/2007/Peru\%20Informe\%20CNAA_2007.pdf

Aponte H. \& D.W. Ramirez. 2011. Humedales de la costa central del Perú: estructura y amenaza de sus comunidades vegetales. Ecología aplicada 10(1):31-39.

Aponte H. \& A. Cano. 2013. Estudio florístico comparativo de seis humedales de la costa central del Perú: Actualización y nuevos retos para su conservación. Revista Latinoamericana de Conservación 3(2):15-27.

Aponte H. \& D. Ramírez. 2014. Riqueza florística y estado de conservación del Área de Conservación Regional Humedales de Ventanilla, Callao, Perú. The Biologist 12(2):270-282.

Batzer D.P., \& R.R. Sharitz (2006). Ecology of Freshwater and Estuarine Wetlands. Los Angeles, USA: University of California Press, Ltd.

Cano A., M.I. La Torre, B. León, J. Roque \& M. Arakaki (1998). Estudio comparativo de la Flora vascular de los Principales Humedales de las Zona Costera del Departamento de Lima, Perú. En A. Cano \& Young (Eds.), Los Pantanos de Villa: Biología y Conservación (pp. 181-190). Lima - Perú: Universidad Nacional Mayor de San Marcos.

Carlos A. 2008. Abundancia poblacional y uso de hábitat de Plegadis ridgwayi "yanavico" en los principales humedales costeros de Lima. Tesis para optar para optar el título profesional de Biólogo con Mención en Zoología. Facultad de Ciencias Biológicas Universidad Nacional Mayor de San Marcos. http://cybertesis.unmsm.edu.pe/handle/cybertesis/912

Di Bitetti M.S. 2012. ¿Qué es el hábitat? Ambiguedad en el uso de Jerga técnica. Ecologia Austral. 22: 137-143.

Expediente Técnico de Establecimiento Zona Reservada Humedales de Puerto Viejo. 2007. Propuesta por el Instituto Nacional de Recursos Naturales-INRENA. 37 pp.

ET-ZRHPV 2009. Expediente técnico de categorización de la Zona Reservada Humedales de Puerto Viejo en Área de Conservación Regional Humedales de Puerto Viejo. 42 pp.

Ferreyra R. 1983. Los tipos de vegetación de la costa peruana. Anales del Jardín Botánico de Madrid. 40(1): 241-256.

Gibbs J.P. 2000. Wetland loss and biodiversity conservation. Conservation Biology. 14: $314-317$.

INRENA 2007. Plan Maestro del Santuario Nacional Los Manglares de Tumbes 2007-2011. Instituto Nacional de Recursos Naturales. Lima, 193 pp.

Iannacone J., J. Mansilla \& K. Ventura. 2003. Macroinvertebrados en las lagunas de Puerto Viejo. Lima, Perú. Ecología Aplicada. 2(1):116-124.

INFORME 2012. Inventario Biológico en la Zona Reservada Humedales de Puerto Viejo-ZRHPV. Contrato No. 071 - 2012 - SERNANP - OA.

INFORME No 01-2016. Evaluación Biológica Rápida de la Zona Reservada Humedales de Puerto Viejo. Anexo No 03-D del Expediente Técnico de Desafectación de la Zona Reservada Humedales de Puerto Viejo. SERNANP. 43 p.
INFORME No 06-2016-SERNANP-ZRHPV/J. Informe de voluntariado sobre el estado de conservación de la ZRHPV. ANEXO No 03-C del Expediente Técnico de Desafectación de la Zona Reservada Humedales de Puerto Viejo. pp 152-156.

INFORME No 638-2016-SERNANP-DDE. Informe que sustenta técnicamente la desafectación de la Zona Reservada $\mathrm{Hu}-$ medales de Puerto Viejo. ANEXO No 03 del Expediente Técnico de Desafectación de la Zona Reservada Humedales de Puerto Viejo. pp 119-134.

Kusch A., J. Cárcamo \& H. Gómez. 2008. Aves acuáticas en el humedal urbano de Tres Puentes, Punta Arenas (53 S), Chile Austral. Anales Instituto Patagonia (Chile), 36(2):45-51.

La Torre M.I. \& H. Aponte. 2009. Flora vascular y vegetación de los humedales de Puerto Viejo. Revista Peruana de Biología. 16(2):215-217. http://dx.doi.org/10.15381/rpb.v16i2.209

Mazuelos Bellido A. 2004. Soft Law: ¿Mucho ruido y pocas nueces? Revista electrónica de estudios internacionales (REEI), (8), $1-40$.

Moschella P. 2012. Variación y protección de humedales costeros frente a procesos de urbanización: casos Ventanilla y Puerto Viejo. Tesis para optar el título de Magíster en Desarrollo Ambiental. Escuela de Posgrado, Pontificia Universidad Católica del Perú. http://tesis.pucp.edu.pe/repositorio/ handle/123456789/4527

Montesinos D. \& L. Mondragon. 2013. Flora y vegetación en tres localidades de una cuenca costeńa: río Acarí, provincia de Caravelí (Arequipa, Perú). Zonas Áridas. 15(1): 11-30.

Paredes C., J. Iannacone \& L. Alvarińo. 2007. Biodiversidad de invertebrados de los humedales de Puerto Viejo, Lima, Perú. Neotrop. Helminthol. 1(2):21-30.

Palomino D \& C. Carranza 2008. Estimación del servicio ambiental de captura del CO2 en la flora de los humedales de Puerto Viejo. Revista del Instituto de Investigaciones FIGMMG. 10 (20): 49-59.

Pautrat L. \& J. Riveros 1998. Evaluación de la avifauna de los Pantanos de Villa, Lima. En: A. Cano y K. Young (Eds.) Los Pantanos de Villa: Biología y Conservación. Museo de Historia Natural-UNMSM. Serie de Divulgación 11:85-95.

Ramirez D.W. \& A. Cano. 2010. Estado de la diversidad de la flora vascular de los Pantanos de Villa (Lima - Perú). Revista Peruana de Biología 17(1):111-114. DOI: http://dx.doi. org/10.15381/rpb.v17i1.58

Ramirez D.W., H. Aponte \& A. Cano. 2010. Flora vascular y vegetación del humedal de Santa Rosa (Chancay, Lima). Revista Peruana de Biología 17(1):105-110. DOI: http://dx.doi. org/10.15381/rpb.v17i1.57

RAMSAR (2006). Manual de la Convención de Ramsar: Guía a la convención sobre los humedales (Ramsar, Irán, 1971), cuarta edición. Secretaría de la Convención de Ramsar, Gland, Suiza.

Riffell S.K., K.J. Gutzwiller \& S.H. Anderson. 1996. Does repeated human intrusion cause cumulative declines in avian richness and abundance? Ecological Applications 6: 492-505. http:// dx.doi.org/10.2307/2269386

Smith T. \& R. Smith. 2007. Ecología, sexta edición. Pearson Educación, S.A. Madrid. 776 p.

Semlitsch R.D. \& J.R. Bodie. 1998. Are small, isolated wetlands expendable? Conservation Biology 12:1129-1133. http:// dx.doi.org/10.1046/j.1523-1739.1998.98166.x

Tello A. \& L. Castillo (eds). 2010. Humedales de la Región Lima: Guía de su flora y fauna silvestre. Gobierno Regional de Lima. Primera edición, Lima-Perú. 97pp.

Young K. 1998. El Ecosistema. En: A. Cano y K. Young (Eds.) Los Pantanos de Villa: Biología y Conservación. Museo de Historia Natural-UNMSM. Serie de Divulgación 11:3-20. 